\title{
THE DOMINION OF ISBELL
}

\author{
BY \\ BARRY MITCHELL
}

\begin{abstract}
A well-known characterization of epimorphisms in the category of rings with identity is imitated to give a similar characterization of epimorphisms in the category of small pre-additive categories. From this one deduces Isbell's "Zigzag Theorem" concerning epimorphisms in Cat.
\end{abstract}

If $f: A \rightarrow B$ is a morphism in a category, then the dominion of $f$ is a morphism $u: D \rightarrow B$ which equalizes any pair $B \rightarrow C$ equalized by $f$, such that if $u^{\prime}: D^{\prime} \rightarrow B$ is any other such morphism, then there is a unique morphism $v: D^{\prime} \rightarrow D$ satisfying $v u=u^{\prime}$. Clearly $u$ is a monomorphism if it exists, and taking $u^{\prime}=f$, we see that $f$ factors through $u$. The morphism $f$ is an epimorphism if and only if its dominion is the identity on $B$, and if $f$ is an equalizer, then its dominion is itself. The dominion of $f$ is the same as the equalizer of its cokernel pair, providing that the latter exists, where the cokernel pair of $f$ is the pair of morphisms $\alpha, \beta$ in the pushout diagram

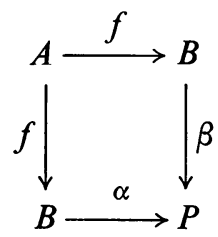

If $f$ factors as an epimorphism followed by a monomorphism, then its dominion is that of the monomorphism.

Let $U: C \rightarrow \boldsymbol{D}$ be a morphism in Cat, or in other words a functor between small categories. Then $U$ can be factored as $\boldsymbol{C} \rightarrow \boldsymbol{C}^{\prime} \rightarrow \boldsymbol{D}$ where $\boldsymbol{C}^{\prime}$ is the subcategory of $\boldsymbol{D}$ generated by the set theoretic image of $U$ (consisting of compositions of morphisms in the image) and clearly $\boldsymbol{C} \rightarrow \boldsymbol{C}^{\prime}$ is an epimorphism. Hence for the purpose of studying its dominion, we may assume that $U$ is the inclusion of a subcategory. In [6] Isbell proved the following remarkable theorem.

ZigZAG TheOREM. Let $\boldsymbol{C}$ be a subcategory of a small category $\boldsymbol{D}$. Then a morphism $s \in \boldsymbol{D}$ is in the dominion of $\boldsymbol{C}$ in $\boldsymbol{D}$ if and only if for some $m \geqq 1$ there is a commutative diagram in $\boldsymbol{D}$ (where all arrows are directed downwards)

Received by the editors April 19, 1971.

AMS 1970 subject classifications. Primary 18A20; Secondary 16A08, $20 \mathrm{M} 25$.

Key words and phrases. Category, functor, natural transformation, dominion, epimorphism, zigzag. 


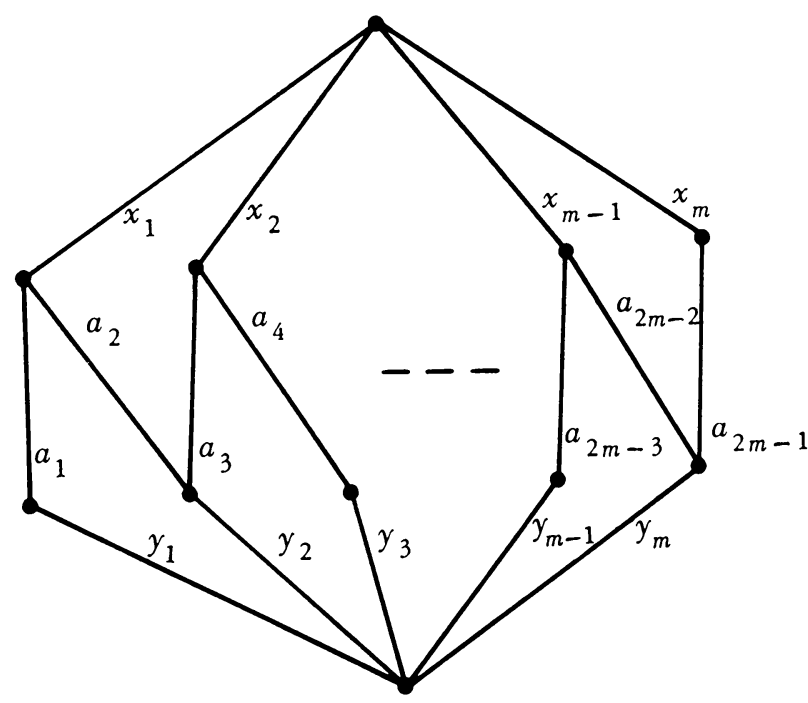

where $x_{1} a_{1}, a_{2 m-1} y_{m}, a_{i} \in C, 1 \leqq i \leqq 2 m-1$, and $x_{1} a_{1} y_{1}=s$.

A commutative diagram (1) in $\boldsymbol{D}$ satisfying the given conditions is called a zigzag of length $m$ in $\boldsymbol{D}$ over $\boldsymbol{C}$ with value $s$. Note that if $m=1$, the condition of commutativity is redundant. It is an amusing exercise to show that the presence of such a zigzag forces two functors from $\boldsymbol{D}$ to agree at $s$ providing they agree on $\boldsymbol{C}$. Isbell proved the other direction by giving an explicit description of the cokernel pair of the inclusion $\boldsymbol{C} \rightarrow \boldsymbol{D}$. However the details involved in this attack appear to be somewhat difficult to write down.

In this note, we shall describe dominions in the category Addcat of all small (pre-)additive categories. This amounts to a straightforward imitation of the simple and elegant proof of Silver [8] characterizing epimorphisms in the category of rings with identity, and would not be worth recording if it were not for the fact that it yields the zigzag theorem as a corollary. The morphisms $a_{i}$ of the zigzag correspond to the passage of scalars through a certain tensor product sign. This is just one of several instances where a nonadditive theorem can be deduced easily from the corresponding additive theorem. Another striking example is Freyd's proof [3] of the nonadditive Hilbert basis theorem-finitely generated commutative monoids are finitely related (due originally to Redei).

In $\$ 1$ we recall how to tensor covariant and contravariant abelian group valued functors, and show how this is used to construct additive Kan extensions relative to an additive functor $U$ ("covariant $U$-extensions" in the terminology of Cartan and Eilenberg). In $\$ 2$ we give two lemmas on adjoints, one of which shows how the problem of finding dominions can sometimes be handled by a change of categories. In $\S 3$ we give the description of the dominion of an additive functor and show how to deduce the zigzag theorem from it. The zigzag theorem for "categories without identities" is obtained in $\S 4$ so as to deduce the original zigzag theorems of Isbell 
[5] and Howie and Isbell [4] for semigroups. Finally, in $\$ 5$ we consider an additive functor $U$ between small categories, and we derive a necessary and sufficient condition that the induced functor $\mathscr{A}^{U}$ on functor categories be faithful (respectively, full) for all additive categories $\mathscr{A}$. Actually this last can be done (as can a large part of the rest of the paper) in the more general setting of closed categories, and unpublished details have been written down by $\mathrm{R}$. Paré. However the relative understandability of the $\mathrm{Ab}$ case seems to justify its special treatment. This treatment can be imitated to give the nonabelian (Ens) case as well, and also the case of $K$-categories (associative $K$-algebras in the one object case) where $K$ is a fixed commutative ring. We do not know if the principal Theorem 3.2 is a theorem about closed categories. Certainly the proof given here depends on additivity.

1. Preliminaries. Composition $f g$ of morphisms means first $f$ and then $g$, but we shall not worry too much about where we place arguments of functions. If $\boldsymbol{C}$ is a category then $|\boldsymbol{C}|$ is its class of objects, and if $p, q \in|\boldsymbol{C}|$, then $\boldsymbol{C}(p, q)$ is the set of morphisms from $p$ to $q$.

By an additive category we understand a category $\mathscr{C}$ equipped with abelian group structures for the sets $\mathscr{C}(p, q)$ such that composition is bilinear. If $\mathscr{C}$ has just one object, then it is just a ring with identity. If $\mathscr{A}$ and $\mathscr{C}$ are additive categories with $\mathscr{C}$ small, then $\mathscr{A}^{\mathscr{C}}$ denotes the category of additive functors from $\mathscr{C}$ to $\mathscr{A}$, where $\mathscr{A}^{\mathscr{C}}(F, G)$ is the abelian group of natural transformations from $F$ to $G$. When $\mathscr{C}$ is a ring, $A b^{\mathscr{C}}$ is the category of right $\mathscr{C}$-modules and $A b^{\mathscr{C} \text { op }}$ is the category of left $\mathscr{C}$-modules.

If $\mathscr{C}$ and $\mathscr{C}^{\prime}$ are additive categories, we let $\mathscr{C} \otimes \mathscr{C}^{\prime}$ denote the additive category whose class of objects is $|\mathscr{C}| \times\left|\mathscr{C}^{\prime}\right|$, where the abelian group of morphisms from $\left(p, p^{\prime}\right)$ to $\left(q, q^{\prime}\right)$ is $\mathscr{C}(p, q) \otimes \mathscr{C}^{\prime}\left(p^{\prime}, q^{\prime}\right)$. Here and elsewhere, unadorned tensor means tensor product over the integers. Composition is defined by

$$
\left(s \otimes s^{\prime}\right)\left(t \otimes t^{\prime}\right)=s t \otimes s^{\prime} t^{\prime} .
$$

Then an additive functor from $\mathscr{C} \otimes \mathscr{C}^{\prime}$ to $\mathscr{A}$ is what is usually called an additive bifunctor $\mathscr{C} \times \mathscr{C}^{\prime} \rightarrow \mathscr{A}$, and if $\mathscr{C}$ and $\mathscr{C}^{\prime}$ are small, then we have isomorphisms of categories

$$
\left(\mathscr{A}^{\mathscr{C}}\right)^{\mathscr{C}^{\prime}}=\mathscr{A}^{\mathscr{C} \otimes \mathscr{C}^{\prime}}=\left(\mathscr{A}^{\mathscr{C}^{\prime}}\right)^{\mathscr{C}} .
$$

Let $\mathscr{C}$ be a small additive category, and let $M \in \mathrm{Ab}^{\mathscr{C}}, \lambda \in \mathscr{C}(p, q), x \in(p) M$. Then in keeping with module notation, we write $x \lambda$ in place of $(x)(\lambda) M$. Likewise if $N \in \mathrm{Ab}^{\mathscr{C} \text { op }}, \lambda \in \mathscr{C}(p, q), y \in N(q)$, then we write $\lambda y$ in place of $N(\lambda)(y)$. Thus $x \lambda$ is an element of $(q) M$, and $\lambda y$ is an element of $N(p)$. We define

$$
M \otimes_{\mathscr{C}} N=\left[\bigoplus_{p \in|\mathscr{C}|}(p) M \otimes N(p)\right] / K
$$

where $K$ is the subgroup of the numerator generated by elements of the form $x \lambda \otimes y-x \otimes \lambda y$. Then $\otimes_{\mathscr{C}}$ is an additive bifunctor to abelian groups. When $\mathscr{C}$ is a ring, $\otimes_{\mathscr{C}}$ is the usual tensor product of right $\mathscr{C}$-modules with left $\mathscr{C}$-modules. 
If $M$ and $N$ are bifunctors, then so is $M \otimes_{\mathscr{C}} N$. For example, if $M \in \mathrm{Ab}^{\mathscr{E} \circ \otimes \mathscr{C}}$ and $N \in \mathrm{Ab}^{\mathscr{C O D} \otimes \mathscr{D}}$, then $M \otimes_{\mathscr{C}} N$ is the bifunctor $\mathscr{E}^{\mathrm{op}} \otimes \mathscr{D} \rightarrow \mathrm{Ab}$ whose value at $(e, d)$ is $M(e,) \otimes_{\mathscr{C}} N(, d)$. This is, of course, a special case of a general principle whereby the values of functors pick up arguments when arguments are added to the domains.

Proposition 1.1. If $F \in \mathrm{Ab}^{\mathscr{E}^{\mathrm{op}} \otimes \mathscr{C}}, G \in \mathrm{Ab}^{\mathscr{C} \mathrm{op} \otimes \mathscr{D}}$, and $H \in \mathrm{Ab}^{\mathscr{E}^{\mathrm{op}} \otimes \mathscr{D}}$, then there is an isomorphism

$$
\mathrm{Ab}^{\mathscr{E} \mathrm{OP} \otimes \mathscr{D}}\left(F \otimes_{\mathscr{C}} G, H\right) \approx \mathrm{Ab}^{\mathscr{E P} \otimes \mathscr{C}}\left(F, \mathrm{Ab}^{\mathscr{D}}(G, H)\right)
$$

which is natural in $F, G$, and $H$.

This is the obvious generalization of Proposition 2.2, p. 165 of Cartan and Eilenberg, and we shall (as did they) leave the proof to the reader. It can be established directly by writing down arrows in both directions and showing that both compositions are identities.

Another natural isomorphism which we shall need is

$$
F \otimes_{\mathscr{C}} \mathscr{C}(, q) \approx F(q)
$$

for $F \in \mathrm{Ab}^{\mathscr{C}}$. It can either be established directly, or it can be deduced from Proposition 1.1 using the Yoneda lemma. Note that in the ring case, this is just the natural isomorphism

$$
M \otimes_{R} R \approx M
$$

relative to a right $R$-module $M$. The fact that (1) is natural in $q$ as well as $F$ corresponds to the fact that (2) is an isomorphism of $R$-modules, not just of abelian groups.

Now let $U: \mathscr{C} \rightarrow \mathscr{D}$ be an additive functor between small additive categories, and take $G$ to be $\mathscr{D}(U(), \quad)$ in Proposition 1.1. By the Yoneda lemma, we have

$$
\mathrm{Ab}^{\mathscr{D}}(\mathscr{D}(U(),), H) \approx H(, U()) .
$$

Thus by Proposition 1.1 we see that

$$
\otimes_{\mathscr{C}} \mathscr{D}(U(), \quad): \mathrm{Ab}^{\mathscr{8} \mathrm{OP} \otimes \mathscr{C}} \rightarrow \mathrm{Ab}^{\mathscr{E}^{\circ \mathrm{P}} \otimes \mathscr{D}}
$$

is a left adjoint for the functor which composes with $U$. If $F \in \mathrm{Ab}^{\mathscr{E}} \otimes \mathscr{Q}$, then $F \otimes_{\mathscr{C}} \mathscr{D}(U(), \quad)$ is called the additive Kan extension of $F$ along $U$. The adjunction morphism

$$
\eta_{F}: F \rightarrow F \otimes_{\mathscr{C}} \mathscr{D}(U(), U())
$$

is giving by identifying $F$ with $F \otimes_{\mathscr{C}} \mathscr{C}$ via the isomorphism (1), and then using the natural transformation of 2 variable functors

$$
\mathscr{C} \rightarrow \mathscr{D}(U(), U())
$$


induced by $U$. Thus when $U$ is full and faithful, $\eta_{F}$ is an isomorphism. In other words, the diagram

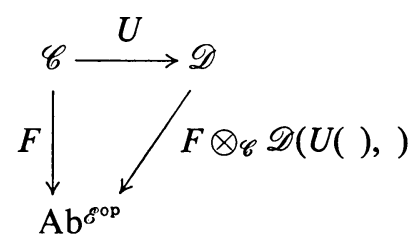

commutes up to natural isomorphism, and so in this case the Kan extension is indeed an extension, up to natural isomorphism. If $U$ is the inclusion of a full subcategory, then by changing some of the values of $F \otimes_{\mathscr{C}} \mathscr{D}(U()$, ), if necessary, we see that $F$ has an extension to $\mathscr{D}$ in the strict sense. This remark will be used in the following section.

The other adjunction

$$
\varepsilon_{H}: H(, U()) \otimes_{\mathscr{C}} \mathscr{D}(U(), \quad) \rightarrow H
$$

is given by $\varepsilon_{H}(x \otimes s)=x s$. In particular, when $\mathscr{E}=\mathscr{D}$ and $H=\mathscr{D}$, this is the morphism

$$
m: \mathscr{D}(, U()) \otimes_{\mathscr{C}} \mathscr{D}(U(), \quad) \rightarrow \mathscr{D}
$$

defined by composition of morphisms in $\mathscr{D}$. The morphism $m$ will play a fundamental role in the sequel.

Everything said above can be repeated in the nonadditive situation. In this case Ens replaces $A b$, cartesian products replace tensor products over the integers, and disjoint unions replace direct sums. For example, if $\boldsymbol{C}$ is a small (nonadditive) category, then relative to $M \in \mathrm{Ens}^{C}$ and $N \in \mathrm{Ens}^{C^{\mathrm{op}}}$ we define

$$
M \times{ }_{C} N=\left[\bigcup_{p \in|C|}(p) M \times N(p)\right] / \sim
$$

where the union is disjoint, and where $\sim$ is the equivalence relation generated by $(x \lambda, y) \sim(x, \lambda y), \lambda \in C(p, q), x \in(p) M, y \in N(q)$.

If $X$ is a set, then $Z X$ denotes the free abelian group on $X$. We can regard $Z:$ Ens $\rightarrow \mathrm{Ab}$ as a functor in the obvious way, called the "addification" functor. Likewise, if $\boldsymbol{C}$ is a nonadditive category, then we can form its addification $\boldsymbol{Z C}$. The objects are those of $\boldsymbol{C}$, and $\boldsymbol{Z C}(p, q)$ is the free abelian group on $\boldsymbol{C}(p, q)$. Composition is defined in the unique way so as to be bilinear and so as to make the inclusion of $\boldsymbol{C}$ in $\boldsymbol{Z C}$ a functor. When $\boldsymbol{C}$ is a monoid, $\boldsymbol{Z C}$ is the usual monoid ring with integer coefficients. Relative to an additive category $\mathscr{A}$ we have an isomorphism of categories $\mathscr{A}^{C}=\mathscr{A}^{z C}$ where the left side is all functors from $C$ to $\mathscr{A}$, whereas the right side is all additive functors from $Z C$ to $\mathscr{A}$. This shows in particular that $Z:$ Cat $\rightarrow$ Addcat is a left adjoint for the functor which forgets additivity. If $M \in \mathrm{Ens}^{C}$, we define

$$
Z M \in \mathrm{Ab}^{C}=\mathrm{Ab}^{Z C}
$$


to be the image of $M$ under the induced functor Ens ${ }^{C} \rightarrow \mathrm{Ab}^{C}$. If $M \in \mathrm{Ens}^{C}$ and $N \in \mathrm{Ens}^{C^{\mathrm{op}}}$, then we have an isomorphism of abelian groups

$$
Z\left(M \times_{C} N\right) \approx Z M \otimes_{z C} Z N
$$

as is seen by defining morphisms in both directions and showing that both compositions are identities. The image of the basis element $(x, y)$ on the left is the element $x \otimes y$ on the right.

2. Two lemmas on adjoints. Recall that a natural transformation of hom functors $\mathscr{A}(A,) \rightarrow \mathscr{A}\left(A_{1}, \quad\right)$ is a monomorphism if and only if the morphism $A_{1} \rightarrow A$ inducing it is an epimorphism (by definition of epimorphism) and is an epimorphism if and only if $A_{1} \rightarrow A$ is a split monomorphism (evaluate at $A_{1}$ ). From this we obtain the following well-known lemma.

Lemma 2.1. Let $S: \mathscr{B} \rightarrow \mathscr{A}$ be a left adjoint for $T: \mathscr{A} \rightarrow \mathscr{B}$. Then $T$ is faithful (respectively, full) if and only if the adjunction morphism $\varepsilon_{A}: S(T(A)) \rightarrow A$ is an epimorphism (resp., split monomorphism) for all $A$.

Dually, $S$ is faithful (full) if and only if the adjunction $\eta_{B}: B \rightarrow T(S(B)$ ) is a monomorphism (split epimorphism) for all $B$.

Proof. Consider the composition of natural transformations

$$
\mathscr{A}\left(A, A^{\prime}\right) \rightarrow \mathscr{B}\left(T(A), T\left(A^{\prime}\right)\right) \stackrel{\approx}{\rightarrow} \mathscr{A}\left(S(T(A)), A^{\prime}\right)
$$

of set valued functors of $A^{\prime}$. This is a monomorphism for all $A$ if and only if $T$ is faithful, and is an epimorphism for all $A$ if and only if $T$ is full. But the morphism $S(T(A)) \rightarrow A$ inducing it is $\varepsilon_{A}$. Therefore the lemma follows from the remarks preceding it.

Lemma 2.2. Let $S: \mathscr{B} \rightarrow \mathscr{A}$ be a faithful left adjoint for $T: \mathscr{A} \rightarrow \mathscr{B}$. Let $B^{\prime} \rightarrow B$ be any morphism in $\mathscr{B}$, and let $D \rightarrow S(B)$ be the dominion of $S\left(B^{\prime}\right) \rightarrow S(B)$. Then the dominion of $B^{\prime} \rightarrow B$ is given by the morphism $P \rightarrow B$ in the pullback diagram

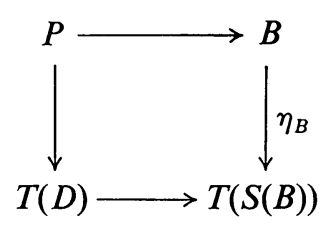

Proof. Since $D \rightarrow S(B)$ is a monomorphism, so is $T(D) \rightarrow T(S(B))$, hence so is $P \rightarrow B$. Let $B^{\prime} \rightarrow B$ equalize $B \rightarrow Y$. Then $S\left(B^{\prime}\right) \rightarrow S(B)$ equalizes $S(B) \rightarrow S(Y)$, hence so does $D \rightarrow S(B)$. Therefore, from the diagram

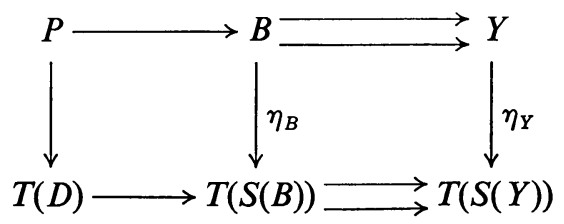


we see that $P \rightarrow B$ equalizes $B \rightarrow{ }_{\rightarrow}^{\rightarrow} \stackrel{\eta_{Y}}{\rightarrow} T(S(Y))$. But $\eta_{Y}$ is a monomorphism by Lemma 2.1. Hence $P \rightarrow B$ equalizes $B \rightarrow Y$.

It remains to be shown that if $Q \rightarrow B$ equalizes any pair $B \rightarrow Y$ which is equalized by $B^{\prime} \rightarrow B$, then $Q \rightarrow B$ factors through $P \rightarrow B$. Let $S\left(B^{\prime}\right) \rightarrow S(B)$ equalize $S(B) \rightarrow X$. Then from the diagram

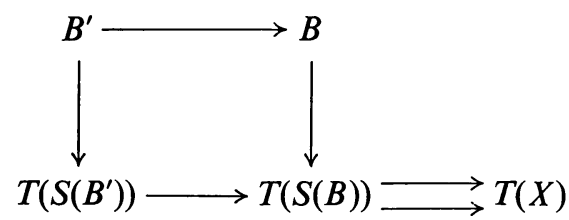

we see that $B^{\prime} \rightarrow B$ equalizes $B \rightarrow T(S(B)) \rightarrow T(X)$. Therefore by assumption so does $Q \rightarrow B$, and so by adjointness it follows that $S(Q) \rightarrow S(B)$ equalizes $S(B) \rightarrow X$. Therefore $S(Q) \rightarrow S(B)$ factors through $D \rightarrow S(B)$, and so by adjointness again we obtain a commutative diagram

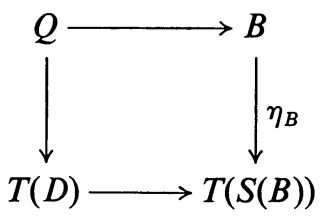

Hence by the pullback property of $P$, we see that $Q \rightarrow B$ factors through $P \rightarrow B$, as required.

We remark that the pullback diagram of Lemma 2.2 is actually an intersection diagram since $\eta_{B}$, as well as $T(D) \rightarrow T(S(B))$, is a monomorphism. The lemma frequently reduces the problem of finding dominions in one category to finding dominions in another. For example, let $\mathscr{R}$ be the category of rings not necessarily with identity, and let $\mathscr{R}^{1}$ be the category of rings with identity. Then the forgetful functor $\mathscr{R}^{1} \rightarrow \mathscr{R}$ has the faithful "adjoin an identity" functor as a left adjoint. Thus the dominion of a morphism $R \rightarrow S$ in $\mathscr{R}$ can be obtained by taking the dominion of $R^{1} \rightarrow S^{1}$ in $\mathscr{R}^{1}$ and intersecting with $S$.

3. Dominions in Addcat. The following lemma reduces the problem of finding the dominion of a morphism $U: \mathscr{C} \rightarrow \mathscr{D}$ in Addcat to the case where $U$ is a surjection on objects. I am indebted to Isbell for the proof.

Lemma 3.1. Let $\mathscr{C} \rightarrow \mathscr{D}$ be a morphism in Addcat and let $\mathscr{D}$ be a full additive subcategory of an additive category $\hat{\mathscr{D}}$. Then the dominions of $\mathscr{C} \rightarrow \mathscr{D}$ and $\mathscr{C} \rightarrow \hat{\mathscr{D}}$ are the same.

Proof. If $s \in \mathscr{D}$, then two functors out of $\hat{\mathscr{D}}$ which distinguish $s$ will also distinguish $s$ when composed with $\mathscr{D} \rightarrow \hat{\mathscr{D}}$. Hence if $s$ is in the dominion of $\mathscr{C} \rightarrow \mathscr{D}$, then it is also in the dominion of $\mathscr{C} \rightarrow \hat{\mathscr{D}}$.

Now suppose that $s$ is in the dominion of $\mathscr{C} \rightarrow \hat{\mathscr{D}}$. Since the value of a functor can always be changed at an object by substituting an isomorphic value without 
altering the other values, we see that the domain and codomain of $s$ must be in the image of $\mathscr{C}$, and so $s$ must be in $\mathscr{D}$ since $\mathscr{D}$ is a full subcategory of $\hat{\mathscr{D}}$. Suppose that $\mathscr{C} \rightarrow \mathscr{D}$ equalizes $F, G: \mathscr{D} \rightarrow \mathscr{E}$, but $F(s) \neq G(s)$. We can compose $F$ and $G$ with the Yoneda imbedding $\mathscr{E} \rightarrow \mathrm{Ab}^{\mathscr{E}^{\circ}}$, and then, as observed in $\S 1$, we can extend to $\hat{F}, \hat{G}: \hat{\mathscr{D}} \rightarrow \mathrm{Ab}^{\mathscr{E}^{\circ \mathrm{p}}}$. Cutting down to a small subcategory of $\mathrm{Ab}^{\mathscr{E}^{\mathrm{op}}}$ containing the images of $\hat{F}$ and $\hat{G}$, we contradict the fact that $s$ is in the dominion of $\mathscr{C} \rightarrow \hat{\mathscr{D}}$.

Any additive functor $U: \mathscr{C} \rightarrow \mathscr{D}$ can be factored as $\mathscr{C} \rightarrow \mathscr{C}^{\prime} \rightarrow \mathscr{D}$ where $\mathscr{C}^{\prime}$ is the additive subcategory generated by the set theoretic image of $U$ (sums of compositions of morphisms in the image of $U$ ) and clearly $\mathscr{C} \rightarrow \mathscr{C}^{\prime}$ is an epimorphism in Addcat. Hence, as in the nonadditive case, we may reduce to the case where $U$ is the inclusion of an additive subcategory in finding its dominion. Actually, for the purpose of the following theorem, we need only assume that $U$ is an injection on objects.

THEOREM 3.2. Let $U: \mathscr{C} \rightarrow \mathscr{D}$ be a morphism of Addcat which is an injection on objects. If $s \in \mathscr{D}(p, q)$, then $s$ is in the dominion of $U$ if and only if $p$ and $q$ are in the image of $U$ and

$$
s \otimes 1_{q}=1_{p} \otimes s \in \mathscr{D}(p, U()) \otimes_{\mathscr{C}} \mathscr{D}(U(), q) .
$$

Proof. By Lemma 3.1 we may assume that $U$ is bijective on objects. Suppose that $F, G: \mathscr{D} \rightarrow \mathscr{E}$ equalize $U$. Then we can define

$$
f: \mathscr{D}(p, U()) \otimes_{\mathscr{C}} \mathscr{D}(U(), q) \rightarrow \mathscr{E}(F(p), G(q))
$$

by the rule $f(r \otimes t)=F(r) G(t)$ where the right side is composition in $\mathscr{E}$. Then we have

$$
f\left(s \otimes 1_{q}\right)=F(s), \quad f\left(1_{p} \otimes s\right)=G(s),
$$

and so if $s \otimes 1_{q}=1_{p} \otimes s$, then $F(s)=G(s)$. Consequently $s$ is in the dominion of $U$.

For the converse, we construct a category $\mathscr{E}$ by taking as its objects those of $\mathscr{D}$, and defining

$$
\mathscr{E}(p, q)=\mathscr{D}(p, q) \times\left[\mathscr{D}(p, U()) \otimes_{\mathscr{C}} \mathscr{D}(U(), q)\right]
$$

where the right side is the product of Abelian groups. Composition is defined, using the bifunctor structure of the second term in the product, by $(s, \alpha)(t, \beta)$ $=(s t, s \beta+\alpha t)$. Then $\mathscr{E}$ is easily seen to be an additive category. We define $F, G$ : $\mathscr{D} \rightarrow \mathscr{E}$ by

$$
F(s)=(s, 0), \quad G(s)=\left(s, s \otimes 1_{q}-1_{p} \otimes s\right) .
$$

Then $F$ and $G$ are additive functors, and if $s$ is in the image of $U$, then $F(s)=G(s)$. On the other hand, if $s \otimes 1_{q} \neq 1_{p} \otimes s$, then $F(s) \neq G(s)$. Hence $s$ is not in the dominion of $U$.

COROllary 3.3. Let $U: \mathscr{C} \rightarrow \mathscr{D}$ be a morphism of Addcat, and suppose that $U$ is an injection on objects. Then $U$ is an epimorphism in Addcat if and only if

$$
m: \mathscr{D}(, U()) \otimes_{\mathscr{C}} \mathscr{D}(U(), \quad) \rightarrow \mathscr{D}
$$

is an isomorphism and $U$ is bijective on objects. 
Proof. If $U$ is an epimorphism, then its dominion is all of $\mathscr{D}$, and so by the theorem $U$ must be bijective on objects and $s \otimes 1_{q}=1_{p} \otimes s$ for all $s \in \mathscr{D}$. Define

$$
n_{p q}: \mathscr{D}(p, q) \rightarrow \mathscr{D}(p, U()) \otimes_{\mathscr{C}} \mathscr{D}(U(), q)
$$

by $n_{p q}(s)=s \otimes 1_{q}$. Then $m_{p q}\left(n_{p q}(s)\right)=s$ and

$$
n_{p q}\left(m_{p q}(s \otimes t)\right)=s t \otimes 1=s(t \otimes 1)=s(1 \otimes t)=s \otimes t .
$$

Hence $m$ is an isomorphism.

Conversely if $U$ is bijective on objects and $m$ is an isomorphism, then, since $m(s \otimes 1)=m(1 \otimes s)=s$, we must have $s \otimes 1=1 \otimes s$. Hence $s$ is in the dominion of $U$ for all $s \in \mathscr{D}$, and so $U$ is an epimorphism.

REMARK 1 . Since the functors $F$ and $G$ constructed in the theorem are identities on objects, the theorem and corollary are valid relative to the category of small additive categories with a fixed set of objects, where morphisms are additive functors preserving objects. In particular, when there is just one object, the corollary is just that of Silver [8] characterizing epimorphisms in the category of rings with identity.

REMARK 2. The category $\mathscr{E}$ constructed in the theorem is not that of the cokernel pair of $U$, as is seen by taking $U$ to be the identity on the ring of integers.

REMARK 3. In $\S 5$ we shall see that the condition that $U$ be an injection on objects is necessary.

The nonadditive version of Theorem 2.2 cannot be obtained by simply imitating the additive proof, since the definition of the category $\mathscr{E}$ and the functors $F$ and $G$ require addition and subtraction of morphisms. To obtain the nonadditive theorem, we consider the additivization functor $Z$ : Cat $\rightarrow$ Addcat which is a faithful left adjoint for the forgetful functor. A morphism $U: C \rightarrow D$ in Cat gives rise to the morphism $\boldsymbol{Z U}: \boldsymbol{Z C} \rightarrow \boldsymbol{Z D}$ in Addcat, relative to which we have, as a special case of the isomorphism (3) of $\S 1$,

$$
\boldsymbol{Z}\left[\boldsymbol{D}(p, U()) \times_{C} \boldsymbol{D}(U(), q)\right] \approx \boldsymbol{Z D}(p, \boldsymbol{Z U}()) \otimes_{z C} \boldsymbol{Z D}(\boldsymbol{Z U}(), q) .
$$

It follows that $\left(s, 1_{q}\right) \sim\left(1_{p}, s\right)$ on the left side if and only if $s \otimes 1_{q}=1_{p} \otimes s$ on the right side. Now by Lemma 2.2, $s$ is in the dominion of $U$ if and only if it in the dominion of $\boldsymbol{Z U}$. Consequently we obtain

Corollary 3.4. Let $U: C \rightarrow D$ be a morphism in Cat which is an injection on objects. If $s \in \boldsymbol{D}(p, q)$, then $s$ is in the dominion of $U$ if and only if $p$ and $q$ are in the image of $U$, and $\left(s, 1_{q}\right) \sim\left(1_{p}, s\right) \in \boldsymbol{D}(p, U()) \times{ }_{C} D(U(), q)$. Hence $U$ is an epimorphism if and only if

$$
m: D(, U()) \times{ }_{C} D(U(), \quad) \rightarrow D
$$

is an isomorphism and $U$ is bijective on objects.

To see how this gives the zigzag theorem, we recall that $\left(s, 1_{q}\right) \sim\left(1_{p}, s\right)$ means that there is a chain 


$$
\begin{aligned}
\left(s, 1_{q}\right) & =\cdots \sim\left(x_{i} a_{2 i}, y_{i}\right)=\left(x_{i+1} a_{2 i+1}, y_{i}\right) \sim\left(x_{i+1}, a_{2 i+1} y_{i}\right) \\
& =\left(x_{i+1}, a_{2 i+2} y_{i+1}\right) \sim \cdots=\left(1_{p}, s\right)
\end{aligned}
$$

with the $a_{i} \in C$. This is easily seen to imply the existence of a zigzag in $\boldsymbol{D}$ over $\boldsymbol{C}$ with value $s$.

If $U: R \rightarrow S$ is a homomorphism of commutative rings with identity, then $S \otimes_{R} S$ in this case is a commutative ring, and is in fact the cokernel pair of $U$ in the category of commutative rings with identity. This makes Theorem 3.2 practically trivial in this case (as observed first, I believe, by H. H. Storrer), and the nonadditive case of commutative monoids can be done independently in exactly the same way. (In fact, what is involved here is undoubtedly a theorem on closed categories.) Thus the zigzag theorem holds also for commutative monoids (Howie and Isbell [4]).

4. Semicategories. The original zigzag theorems of Isbell [5] and Howie and Isbell [4] were for semigroups. Now if one grants an interest in one object categories without identities-i.e., semigroups-then one should grant an interest in several object categories without identities. Thus at the risk of being censured by the mathematical community, let us define a semicategory $C$ to consist of a class of objects $|\boldsymbol{C}|$ together with a set $\boldsymbol{C}(p, q)$ for each $p, q \in|\boldsymbol{C}|$ and an associative composition

$$
C(p, q) \times C(q, r) \rightarrow C(p, r) .
$$

We observe that the sets $\boldsymbol{C}(p, p)$ may be empty. A semifunctor $U: C \rightarrow D$ of semicategories assigns objects $U(p) \in|\boldsymbol{D}|$ to objects $p \in|\boldsymbol{C}|$ and morphisms $U(s)$ $\in \boldsymbol{D}(U(p), U(q))$ to morphisms $s \in \boldsymbol{C}(p, q)$ and preserves composition. The class of small semicategories and semifunctors forms a category Semicat, and we have a forgetful functor Cat $\rightarrow$ Semicat. A faithful left adjoint for this functor assigns to a semicategory $\boldsymbol{C}$ the category $\boldsymbol{C}^{1}$ obtained by adjoining an identity morphism to $C(p, p)$ for each $p \in|C|$, whether or not it already had one. As usual, in studying dominions in Semicat, we may assume that the functor in question is the inclusion of a subsemicategory.

THEOREM 4.1. Let $C$ be a subsemicategory of a small semicategory $D$. If $s \in \boldsymbol{D}-\boldsymbol{C}$, then $s$ is in the dominion of $\boldsymbol{C}$ in $\boldsymbol{D}$ if and only if there is a zigzag in $\boldsymbol{D}$ over $C$ with value $s$.

Proof. By Lemma 2.2, $s$ is in the dominion of $\boldsymbol{C}$ in $\boldsymbol{D}$ if and only if it in the dominion of $\boldsymbol{C}^{1}$ in $\boldsymbol{D}^{1}$. Hence by the zigzag theorem, it suffices to establish the following lemma.

Lemma 4.2. Let $\boldsymbol{C}$ be a subcategory of a category $\boldsymbol{D}$. If $s \notin C$, and if there is $a$ zigzag in $\boldsymbol{D}$ over $\boldsymbol{C}$ with value s, then there is one where (with reference to the diagram (1) of the zigzag theorem) none of the $x_{i}, y_{i}, a_{i}$ are identities.

Proof. Let $m$ be the minimum length of a zigzag in $\boldsymbol{D}$ over $\boldsymbol{C}$ with value $\boldsymbol{s}$. If $m=1$, then none of $x_{1}, a_{1}, y_{1}$ is 1 since otherwise $s \in C$. Thus we may assume $m>1$. 
In this case the lemma follows from the following more detailed lemma, most of which was stated in Howie and Isbell [4, Lemma 1.2].

LemMA 4.3. Let $\boldsymbol{C}$ be a subsemicategory of a semicategory $\boldsymbol{D}$, and suppose that the diagram (1) of the zigzag theorem is a zigzag in $\boldsymbol{D}$ over $\boldsymbol{C}$ of smallest length with value s. Then:

(i) The only compositions of an $x$ with an a or an a with $a y$ which make sense relative to the diagram and which are in $C$ are $x_{1} a_{1}$ and $a_{2 m-1} y_{m}$ (these two being required by definition).

(ii) For no $i, 2 \leqq i \leqq 2 m-1$, are there elements $a^{\prime}, a^{\prime \prime} \in D^{1}$ such that $a^{\prime} a_{i} a^{\prime \prime} \in \boldsymbol{C}$ and

$$
\begin{aligned}
a^{\prime} a_{i} & =a_{i+1}, \\
a_{i} a^{\prime \prime} & =a_{i-1},
\end{aligned} \quad \text { i even; } \quad \begin{aligned}
a^{\prime} a_{i} & =a_{i-1}, \\
a_{i} a^{\prime \prime} & =a_{i+1},
\end{aligned} \quad \text { i odd. }
$$

Proof. (i) If $x_{i} a_{2 i-1} \in C$ for some $i, 2 \leqq i \leqq m$ then we find a zigzag of length $m-i+1$. If $x_{i} a_{2 i} \in C$ for some $i, 1 \leqq i \leqq m-1$, then we find a zigzag of length $m-i$. Dually for the compositions of $a$ 's with $y$ 's.

(ii) The negation of the assertion gives rise to a zigzag of length $m-1$ where one of the $a^{\prime}$ 's is $a^{\prime} a_{i} a^{\prime \prime}$.

Likewise we can form the category Addsemicat of small additive semicategories and the forgetful functor from Addcat has as left adjoint the faithful functor which assigns to $\mathscr{C}$ the additive category $\mathscr{C}^{1}$ where $\left|\mathscr{C}^{1}\right|=|\mathscr{C}|$ and

$$
\begin{aligned}
\mathscr{C}^{1}(p, q) & =\mathscr{C}(p, q), & & p \neq q, \\
& =\mathscr{C}(p, p) \oplus Z, & & p=q .
\end{aligned}
$$

By Lemma 2.2, the dominion of $\mathscr{C} \rightarrow \mathscr{D}$ in Addsemicat is the intersection of $\mathscr{D}$ with the dominion of $\mathscr{C}^{1} \rightarrow \mathscr{D}^{1}$ in Addcat. What is really involved here is, needless to say, a construction in the theory of closed categories.

5. Fullness and faithfulness of $\mathscr{A}^{U}$.

THeOREM 5.1. Let $U: \mathscr{C} \rightarrow \mathscr{D}$ be a morphism in Addcat. Then a necessary and sufficient condition that the induced functor $\mathscr{A}^{U}: \mathscr{A}^{\mathscr{D}} \rightarrow \mathscr{A}^{\mathscr{C}}$ be faithful (resp., full) for all additive categories $\mathscr{A}$ is that the morphism

$$
m: \mathscr{D}(, U()) \otimes_{\mathscr{C}} \mathscr{D}(U(), \quad) \rightarrow \mathscr{D}
$$

be an epimorphism (resp., split monomorphism) in $\mathrm{Ab}^{\mathscr{D}^{\mathrm{op}} \otimes \mathscr{D}}$.

Proof. For necessity, we take $\mathscr{A}$ to be $\mathrm{Ab}^{\mathscr{O}{ }^{\circ p}}$. Then, as was pointed out in $\S 1$, $m$ is the front adjunction evaluated at $\mathscr{D}$ relative to the right adjoint $\mathscr{A}^{U}$. Therefore if $\mathscr{A}^{U}$ is faithful (full), then by Lemma 2.1, $m$ is an epimorphism (split monomorphism).

Now suppose that $m$ is an epimorphism. This is clearly equivalent to the condition that $1_{p} \in \mathscr{D}(p, p)$ be in the image of $m_{p p}$, or in other words that we can write

$$
1_{p}=\sum_{i=1}^{k} s_{i} t_{i}, \quad s_{i} \in \mathscr{D}\left(p, U\left(r_{i}\right)\right), \quad t_{i} \in \mathscr{D}\left(U\left(r_{i}\right), p\right)
$$


for all $p \in|\mathscr{D}|$. Then if $\mathscr{A}$ is any additive category and if $\alpha: F \rightarrow G$ is a morphism in $\mathscr{A}^{\mathscr{D}}$, we can write

$$
\alpha_{p}=\alpha_{p} \sum_{i=1}^{k} G\left(s_{i}\right) G\left(t_{i}\right)=\sum_{i=1}^{k} F\left(s_{i}\right) \alpha_{U\left(r_{i}\right)} G\left(t_{i}\right) .
$$

Hence $\alpha_{p}$ is determined once we know the values $\alpha_{U(r)}, r \in|\mathscr{C}|$, and it follows that $\mathscr{A}^{U}$ is faithful.

Finally, suppose that $m$ is a split monomorphism, and let $n$ be a retraction for it. Let $F, G \in \mathscr{A}^{\mathscr{I}}$, and let $\beta: U F \rightarrow U G$ be a morphism in $\mathscr{A}^{\mathscr{C}}$. Then for $p, q \in|\mathscr{D}|$, we have the diagram

$$
\begin{gathered}
\mathscr{D}(p, U()) \otimes_{\mathscr{C}} \mathscr{D}(U(), q) \underset{n_{p q}}{\stackrel{m_{p q}}{\rightleftarrows}} \mathscr{D}(p, q) \\
\downarrow_{p q} \\
\mathscr{A}(F(p), G(q))
\end{gathered}
$$

where $f_{p q}(s \otimes t)=F(s) \beta_{r} G(t), s \in \mathscr{D}(p, U(r)), t \in \mathscr{D}(U(r), q)$. Then $f, n$, and $m$ are all natural in $p$ and $q$. For $p \in|\mathscr{D}|$, define

$$
\alpha_{p}=f_{p p}\left(n_{p p}\left(1_{p}\right)\right)
$$

By naturality of $n$ and $f$ and the fact that a morphism $s \in \mathscr{D}(p, q)$ can be written as either $1_{p} s$ or $s 1_{q}$, we see that $\alpha$ is a natural transformation from $F$ to $G$. Furthermore, if $r \in|\mathscr{C}|$, then

$$
\alpha_{U(r)}=f\left(n\left(1_{U(r)}\right)\right)=f\left(n\left(m\left(1_{U(r)} \otimes 1_{U(r)}\right)\right)\right)=f\left(1_{U(r)} \otimes 1_{U(r)}\right)=\beta_{r} .
$$

This shows that $\mathscr{A}^{U}$ is full and completes the proof.

Combining the theorem with Corollary 3.3, we obtain:

COROllaRy 5.2. Let $U: \mathscr{C} \rightarrow \mathscr{D}$ be an epimorphism of Addcat which is an injection (and hence a bijection) on objects. Then $\mathscr{A}^{U}$ is full and faithful for all additive categories $\mathscr{A}$.

The corollary answers a question of Isbell [6], who showed that it was true in the case of ring epimorphisms. To see that the condition that $U$ be an injection on objects is necessary, let $\mathbf{2}$ be the totally ordered set of two elements and let $\boldsymbol{N}$ be the monoid of natural numbers, and take $U: \mathbf{2} \rightarrow N$ to be the functor which assigns the generator of $N$ to the nonidentity of 2 . Then $U$ is an epimorphism since the subcategory of $N$ generated by its image is all of $N$. However, if $A$ is any nonzero object of an additive category $\mathscr{A}$, then the endomorphism

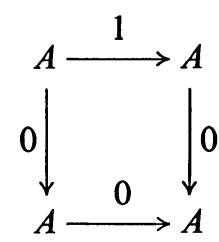

in $\mathscr{A}^{2}$ cannot come from anything in $\mathscr{A}^{N}$. 


\section{REFERENCES}

1. H. Cartan and S. Eilenberg, Homological algebra, Princeton Univ. Press, Princeton, N. J., 1956. MR 17, 1040.

2. A. H. Clifford and G. B. Preston, The algebraic theory of semigroups. Vol. 1, Math. Surveys, no. 7, Amer. Math. Soc., Providence, R. I., 1961. MR 24 \#A2627.

3. P. Freyd, Redei's finiteness theorem for commutative semigroups, Proc. Amer. Math. Soc. 19 (1968), 1003. MR 37 \#2875.

4. J. M. Howie and J. R. Isbell, Epimorphisms and dominions. II, J. Algebra 6 (1967), 7-21. MR 35 \#105b.

5. J. R. Isbell, Epimorphisms and dominions, Proc. Conf. Categorical Algebra (La Jolla, Calif., 1965), Springer, New York, 1966, pp. 232-246. MR 35 \#105a.

6. —, Epimorphisms and dominions. III, Amer. J. Math. 90 (1968), 1025-1030. MR 38 \#5877.

7. — Epimorphisms and dominions. IV, J. London Math. Soc. (2) 1 (1969), 265-273. MR 41 \#1774.

8. L. Silver, Noncommutative localizations and applications, J. Algebra 7 (1967), 44-76. MR 36 \#205.

Department of Mathematics, Dalhousie University, Halifax, Nova Scotia, Canada

Current address: Department of Mathematics, Rutgers University, New Brunswick, New Jersey 08903 